\title{
EVALUACIÓN CUALITATIVA DE LAS DIRECTIVAS DE IGUALDAD RACIAL Y DE IGUALDAD EN EL EMPLEO
}

\section{QUALITATIVE ASSESSMENT OF DIRECTIVES ON RACIAL EQUALITY AND EQUALITY IN EMPLOYMENT}

\author{
Jesús Pérez Viejo \\ Universidad Carlos III. Getafe (Madrid). España/Spain \\ jeperezv@.clio.uc3m.es
}

César Muñoz Martínez

Universidad Nacional de Educación a Distancia. Madrid. España/Spain

cmunoz@,cee.uned.es

Recibido/Received: 15/07/2016

Modificado/Modified: 24/09/2016

Aceptado/Accepted: 2/10/2016

\section{RESUMEN}

El objetivo es evaluar los procesos de transposición en los Estados Miembros de la Unión Europea de la Directiva 2000/43/CE relativa a la aplicación de la igualdad de trato de las personas independientemente de su origen racial o étnico y la Directiva 2000/78/CE relativa al a la igualdad de trato en el empleo y la ocupación. La protección contra la discriminación es uno de los ámbitos en los que la legislación comunitaria afecta más de cerca a los ciudadanos europeos. El marco normativo creado por las dos Directivas de la UE de lucha contra la discriminación ha configurado la legislación europea durante más de una década. Los condicionamientos derivados de los desarrollos normativos afectan a las políticas comunes y estatales y a las intervenciones sociales y modalidades del trabajo social, orientados a la erradicación de las discriminaciones de origen racial y de todas aquellas que se manifiestan en mundo laboral.

\section{PALABRAS CLAVE}

Directivas, transposición, discriminación, trabajo social, empleo.

\section{SUMARIO}

1. Consideraciones metodológicas. 2. Humus originario de las directivas contra la discriminación. 3. Evolución de las precisiones, facultades, transposiciones y factores de incidencia. 4. Tendencias de aplicación y cumplimiento en las escalas estatales. 5. Tendencia normativa y efectos convergentes. 6 . Conclusiones. Bibliografía.

\footnotetext{
ABSTRACT

The aim is to evaluate the process of implementation in the Member States of the European Union Directive 2000/43 / EC of 29 June 2000 on the implementation of equal treatment persons irrespective of racial or ethnic origin and Directive 2000/78 / EC of 27 November 2000 on equal treatment in employment and occupation. Protection against discrimination is one of the areas in which EU
} 
legislation affects closer to European citizens. The regulatory framework created by the two EU directives to combat discrimination has set up European legislation for more than a decade. The constraints arising from regulatory developments affecting the policies of the European Union and its Member States, and social interventions and methods of social work, aimed at the eradication of discrimination of racial origin and all those that manifest themselves in world labor.

\section{KEYWORDS}

Directives, transposition, discrimination, social work, employment.

\section{CONTENTS}

1. About methodology. 2. Humus. 3. Evolution. 4. Tendency. 5. Tendency. 6. Conclusions. References.

\section{CONSIDERACIONES METODOLÓGICAS}

Para desarrollar de esta investigación, hemos empleado un método analítico-cualitativo con enfoque descriptivo, realizando un análisis de contenido de las principales fuentes primarias y secundarias relacionadas con la regulación y la dinámica de las políticas de igualdad. Para ello, se han aplicado distintos métodos, partiendo de la clasificación y definición, previo trabajo de búsqueda y consulta del acervo comunitario y de los fondos documentales que han servido de base y que han sido el instrumento fundamental de este trabajo.

Esta investigación de acción conjuga métodos y procedimientos para, desde un prisma jurídico-social, conocer, primero, y señalar, después, las implicaciones derivadas del marco normativo creado por las Directivas de la EE en la lucha contra la discriminación.

\section{HUMUS ORIGINARIO DE LAS DIRECTIVAS CONTRA LA DISCRIMINACIÓN}

Aunque el término legislación europea contra la discriminación parece indicar la existencia de un sistema normativo paneuropeo en materia de no discriminación, en realidad es resultado de la combinación de diversos marcos (Bamforth et al., 2008).

La prohibición de la discriminación está recogida en el artículo 14 del Convenio Europeo de Derechos Humanos (CEDH), que garantiza la igualdad de trato en el disfrute de los restantes derechos y libertades reconocidos en el mismo. El Protocolo 12 (2000) al CEDH, adoptado en Roma el 4 de diciembre del año 2000, amplía el alcance de la prohibición a la discriminación al garantizar la igualdad de trato en el disfrute de todos los derechos derivados de la legislación nacional de los países firmantes. De hecho, en la Exposición de Motivos se explica que la adopción del Protocolo responde al deseo de reforzar la protección contra la discriminación, considerada un elemento fundamental de la garantía de los derechos humanos. El referido Protocolo surgió en un contexto fuertemente influenciado por la implementación de políticas que tuviesen por objeto la igualdad racial y de género.

Durante la década de 1990, los grupos de interés público presionaron con fuerza en favor de la ampliación de la prohibición de la discriminación a otras áreas como la raza y el origen étnico, así como la orientación sexual, las creencias religiosas, la edad y la discapacidad. Los temores al resurgimiento del nacionalismo extremista en algunos Estados miembros de la Unión estimularon la voluntad política de los dirigentes hacia la modificación del Tratado de la Comunidad Europea para atribuir a la Comunidad competencias legislativas en estas áreas. 
Fruto de esta atribución, en el año 2000 se adoptan dos directivas: la Directiva de igualdad en el empleo que prohibía la discriminación por motivos de orientación sexual, creencias religiosas, edad y discapacidad en el área del empleo; y la Directiva de igualdad racial que prohibía la discriminación por motivos de raza y origen étnico en el ámbito del empleo, pero también en el acceso al sistema de bienestar social y a la seguridad social, así como a los bienes y servicios. Esta importante ampliación del alcance de la legislación comunitaria contra la discriminación responde a la convicción de que para que las personas puedan desarrollar todo su potencial en el mercado laboral resulta esencial garantizar la igualdad de acceso en áreas como la salud, la educación y la vivienda.

En 2004, la Directiva de igualdad de trato entre hombres y mujeres en el acceso a los bienes y servicios amplió el ámbito de la discriminación por razón de género al área de los bienes y servicios. No obstante, la protección por motivos de género no alcanza el grado de protección reconocido en la Directiva de igualdad racial, pues la Directiva de igualdad entre hombres y mujeres en materia de seguridad social garantiza la igualdad de trato únicamente en relación con la seguridad social y no con el sistema general de bienestar social, como la protección social y el acceso a la asistencia sanitaria y la educación.

Aunque la orientación sexual, las creencias religiosas, la discapacidad y la edad sólo son motivos protegidos en el ámbito del empleo, las instituciones de la Unión están analizando actualmente una propuesta, denominada como la "Directiva Horizontal", para ampliar la protección por estos motivos al ámbito del acceso a los bienes y servicios.

Los tratados originales de las Comunidades Europeas no incluían ninguna referencia a los derechos humanos y su protección, pues no se consideraba que la creación de un área de libre comercio en Europa pudiera tener repercusiones relevantes en materia de derechos humanos. Sin embargo, a medida que comenzaron a plantearse demandas ante el Tribunal de Justicia de la Unión Europea relativas a asuntos en los que se alegaban incumplimientos de los derechos humanos por parte de la legislación comunitaria, el Tribunal desarrolló un corpus jurisprudencial conocido como los «principios generales» del Derecho Comunitario.

Según el Tribunal, estos principios generales reflejan el contenido de la protección de los derechos humanos de los distintos ordenamientos jurídicos estatales y de los tratados de derechos humanos, en particular del CEDH. El TJUE manifestó así su voluntad de garantizar el ajuste del marco jurídico comunitario a estos principios.

Reconociendo que sus políticas pueden afectar a los derechos humanos y con el propósito de hacer que los ciudadanos se sientan más "próximos" a la UE, ésta y sus Estados miembros promulgaron en el año 2000 la Carta de Derechos Fundamentales de la Unión Europea, que contiene una lista de derechos humanos inspirada en los derechos contenidos en las constituciones de los Estados miembros, el CEDH y los tratados universales de derechos humanos, como la Convención de las Naciones Unidas sobre los Derechos del Niño. En la versión aprobada en 2000, la Carta tenía un carácter meramente "declarativo" y, por tanto, jurídicamente no vinculante, aunque la Comisión Europea manifestó que sus propuestas legislativas se ajustarían a la misma.

Desde la entrada en vigor del Tratado de Lisboa, en 2009, la Carta modificó su naturaleza y adquirió carácter jurídicamente vinculante, lo que la hace de obligado cumplimiento tanto para las instituciones comunitarios como para los Estados miembros, si bien, en el caso de estos últimos, únicamente en la aplicación de la legislación europea. El artículo 21 de esta Carta prohíbe la discriminación por diversos motivos, lo que significa que los ciudadanos europeos tienen la potestad de reclamar si consideran que la legislación comunitaria o estatal, resultante de la aplicación de la anterior, no respetan la Carta de Derechos Fundamentales. En paralelo, también los órganos jurisdiccionales nacionales pueden solicitar orientación 
sobre la correcta interpretación de la normativa de la Unión al TJUE, a través del procedimiento de cuestión prejudicial previsto en el artículo 267 del Tratado de Funcionamiento de la Unión Europea.

Las normativas de la UE y el CEDH se encuentran estrechamente vinculadas. Todos los Estados miembros se han incorporado al Convenio y, como ya se ha indicado, el TJUE se inspira en el CEDH para determinar el alcance de la protección de derechos humanos con arreglo a la normativa comunitaria. La Carta de Derechos Fundamentales refleja también la gama de derechos del Convenio, si bien es cierto que no se limita únicamente a ellos. Por tanto, aunque la UE no es propiamente miembro del $\mathrm{CEDH}$, su normativa se ajusta en gran medida al Convenio. No obstante, si una persona desea formular una reclamación en materia de incumplimiento de los derechos humanos por parte de la UE, no puede llevarla ante el TEDH, sino que debe optar entre acudir a los tribunales nacionales, que podrán remitir posteriormente el caso al TJUE a través del procedimiento de cuestión prejudicial, o reclamar indirectamente contra la UE ante el TEDH, demandando a un Estado miembro.

En consonancia, el Tratado de Lisboa contiene una disposición que obliga a la UE a incorporarse como parte por derecho propio al CEDH, cuyo Protocolo 14 modifica el Convenio para hacer posible esta incorporación. Aunque no está aún claro cuál será el efecto práctico de esta disposición $\mathrm{y}$, en particular, cuál será la relación futura entre el TJUE y el TEDH, pues las negociaciones para la adhesión de la UE pueden extenderse durante años, al menos permitirá a los ciudadanos demandar directamente a la UE ante el TEDH por el incumplimiento del CEDH.

La protección contra la discriminación en Europa puede encontrarse tanto en la normativa de la Unión como en el CEDH. Aunque estos dos sistemas son complementarios en gran medida y se refuerzan mutuamente, existen algunas diferencias, que los profesionales del Derecho deben conocer.

El CEDH protege a todas las personas situadas bajo la jurisdicción de sus cuarenta y siete Estados parte, mientras que las directivas comunitarias contra la discriminación sólo ofrecen protección a los ciudadanos de los veintisiete Estados miembros. El artículo 14 del CEDH prohíbe la discriminación únicamente en relación con el ejercicio de otros derechos reconocidos en el Tratado, mientras que el Protocolo 12 confiere carácter independiente a la prohibición de la discriminación.

En la normativa comunitaria contra la discriminación, la prohibición de la discriminación es de naturaleza autónoma, pero está limitada a ámbitos concretos, como el empleo. Las instituciones europeas están jurídicamente obligadas a cumplir la Carta de Derechos Fundamentales de la Unión, incluidas sus disposiciones sobre no discriminación y simultáneamente los Estados miembros de la Unión deben cumplir también la Carta al aplicar el derecho comunitario.

\section{EVOLUCIÓN DE LAS PRECISIONES, FACULTADES, TRANSPOSICIONES Y FACTORES DE INCIDENCIA}

Algunos Estados miembros apenas contaban con legislación en este ámbito antes de la transposición de las dos Directivas, de modo que éstas han introducido nuevos elementos, como la protección contra la discriminación por razones de edad, en las legislaciones de todos los Estados miembros (Besson, 2006).

Las Directivas contra la discriminación son dos referencias normativas de máxima importancia para el ámbito del trabajo social y extensible a todas sus modalidades de 
intervención, debido a que los procesos de transposición llevados a cabo por los parlamentos nacionales para convertirlas en normas de ámbito estatal, no siempre tienen como resultado que la norma nacional resultante conserve las capacidades y precisiones pretendidas por la norma europea, y ello constituye parte de las causas principales para que la normativa europea sea evaluada y perfeccionada periódicamente. La trascendencia normativa de estas dos directivas, radican en las siguientes facultades:

Prohíben la discriminación por motivos de origen racial o étnico (Directiva 2000/43/CE), religión o convicciones, discapacidad, edad u orientación sexual (Directiva 2000/78/CE).

Proporcionan protección en ámbitos clave de la vida: el empleo y la formación profesional (ambas Directivas); la educación, la seguridad social y la asistencia sanitaria, y la oferta de bienes y servicios y el acceso a los mismos, incluida la vivienda (Directiva 2000/43/CE).

Prohíben distintas formas de discriminación: la discriminación directa o indirecta, el acoso, el mandato de discriminación y la victimización;

Exigen a los Estados miembros que dispongan sanciones y recursos eficaces.

Las evaluaciones de la aplicación de ambas Directivas fueron adoptadas en 2006 y en 2008 respectivamente. Dado que ambas Directivas deben ser objeto de informes periódicos y que el enfoque reglamentario y el contenido de la mayoría de las disposiciones son idénticos, se presentó un informe evaluador conjunto. Además, la mayoría de los Estados miembros han transpuesto las dos Directivas en un único acto jurídico nacional. En este punto, es importante reseñar que los primeros informes se adoptaron cuando la mayoría de Estados miembros acababan de transponer las dos Directivas en sus ordenamientos jurídicos y, por consiguiente, carecían de experiencia en su aplicación.

En la actualidad, los veintiocho Estados miembros han transpuesto las Directivas y han adquirido experiencia en su aplicación. El Tribunal de Justicia de la Unión Europea (TJUE) ha desarrollado asimismo la interpretación de las Directivas a través de su jurisprudencia (Chalmers et al., 2010).

En este punto se va a realizar una aproximación cualitativa a la evaluación de la aplicación de dos Directivas, a fin de conocer la interpretación formulada por el TJUE y por los órganos jurisdiccionales nacionales, con el objetivo de identificar las contingencias sociales futuras que deben afrontar las capacidades que ostentan estas normas del acervo europeo derivado.

Las dos Directivas han sido transpuestas a las legislaciones nacionales en los veintiocho Estados miembros y la Comisión Europea ha sido el órgano garante de controlar que las leyes estatales se atuviesen a las disposiciones de las Directivas. Así pues, se han incoado procedimientos de infracción por falta de conformidad contra veinticinco Estados miembros, principalmente entre 2005 y 2007.

Una proporción importante de los países, experimentaron, en una fase inicial, problemas en la adaptación de la normativa comunitaria a sus ordenamientos debido a la novedad jurídica que suponían los preceptos de ambos Directivas. Los problemas más comunes que se plantearon, se relacionaban con el contenido de las definiciones de discriminación directa e indirecta, acoso, victimización y legitimación procesal de las organizaciones interesadas.

La Directiva 2000/43/CE hubo de transponerse antes del 19 de julio de 2003 por los Estados miembros de la UE-15; antes del 1 de mayo de 2004, por los Estados de la UE-10; antes del 1 de enero de 2007, por Rumanía y Bulgaria, y antes del 1 de julio de 2013, por Croacia. La transposición de la Directiva 2000/78/CE hubo de ser completada antes del 2 de diciembre de 2003 por los Estados de la UE-15 y en los mismos plazos antes indicados por los nuevos Estados miembros. Con todo, la Directiva 2000/78/CE preveía hasta tres años adicionales para transponer las disposiciones relativas a la edad y a la discapacidad. La 
mayoría de los procedimientos de infracción de primera generación han sido archivados debido a que los Estados miembros han adaptado su legislación a las Directivas.

Uno de los procedimientos de infracción incoados por la Comisión dio lugar a una sentencia del TJUE, que declaraba que el Estado miembro había incumplido su obligación de transponer correctamente la Directiva 2000/78/CE en lo relativo a la obligación de realizar ajustes razonables para las personas con discapacidad en el lugar de trabajo.

La Comisión Europea realiza una supervisión continuada de la evolución de la situación en los Estados miembros e incoa procedimientos de infracción cuando es necesario. Las instancias comunitarias reciben diversas denuncias al año en referencia a estas Directivas una media de veinte a treinta aproximadamente, pero la mayoría de ellas son relativas a casos individuales de discriminación, que no atañen a la transposición o aplicación incorrecta de las Directivas y, por consiguiente, no dan lugar a procedimientos de infracción.

En el ámbito nacional se tramita un número considerablemente mayor de denuncias. Pese a que los recursos previstos para los casos individuales de discriminación únicamente se pueden presentar con arreglo al Derecho nacional y solo pueden invocarse ante los órganos jurisdiccionales nacionales, es tarea de la Comisión examinar si una denuncia determinada revela una incorrecta transposición o aplicación de las Directivas por parte del Estado miembro de que se trate. En lo que respecta a los procedimientos de infracción, están pendientes tres incoados por denuncias con arreglo a la Directiva 2000/78/CE.

\section{TENDENCIAS DE APLICACIÓN Y CUMPLIMIENTO EN LAS ESCALAS ESTATALES}

Las Directivas han sido transpuestas a las legislaciones de los Estados miembros, pero las experiencias nacionales ponen de relieve que aún subsisten problemas en lo que se refiere a su aplicación y ejecución.

En esta línea, la Comisión Europea, la Red europea de organismos de promoción de la igualdad (EQUINET), la Agencia de Derechos Fundamentales de la Unión Europea y todos los Estados miembros a título individual han publicado orientaciones pertinentes para la aplicación de las dos Directivas con objeto de solucionar esos problemas. Además, la Comisión Europea financia la formación de jueces y otros profesionales del Derecho, con el objetivo de fomentar la correcta aplicación de las Directivas mediante un mejor conocimiento de la legislación europea sobre la igualdad de trato.

Las Directivas destacan la importancia de la difusión de información para garantizar que los interesados conozcan sus derechos en materia de igualdad de trato, pues gran parte de los ciudadanos desconocen sus derechos en este ámbito. Todos los europeos, no solo las minorías, a menudo desconocen sus derechos.

A título de ejemplo, suele ignorarse que la discriminación laboral está prohibida incluso en la fase de solicitud de un puesto de trabajo. Muchos Estados miembros consideran ese desconocimiento un problema importante y han informado sobre distintas formas de solucionarlo: documentos de información y de orientación, campañas de concienciación y portales de información, en particular para grupos específicos -minorías, jóvenes-, etc.

Una parte considerable de los Estados miembros remarcan que la financiación de "EU Progress" se ha empleado para realizar proyectos de concienciación. Esta fue también una de las recomendaciones presentadas por la Agencia de los Derechos Fundamentales de la UE, en su Dictamen 1/2013 sobre las dos Directivas. Dirigirse a las personas que están expuestas en mayor medida a situaciones de discriminación, así como a las que se encuentran en 
posición de cometer las infracciones, tales como los empresarios, se revela como un empleo eficaz de los recursos.

En el ámbito del empleo, los sindicatos y los interlocutores sociales desempeñan un papel clave en la concienciación de los trabajadores y empresarios en la lucha contra la discriminación. La mayoría de Estados miembros también ofrecen orientaciones prácticas útiles sobre sus leyes contra la discriminación en el lugar de trabajo.

Las Directivas no obligan a los Estados miembros a recabar datos sobre la igualdad de trato. No obstante, la recogida y el análisis son responsabilidad de los Estados miembros y contribuyen a la lucha contra la discriminación y a la promoción de la igualdad, permitiendo aportar pruebas de discriminación existente, poniéndola de manifiesto y cuantificándola.

La escasez de datos sobre la igualdad dificulta la evaluación de las situaciones y la prueba de su existencia. Esto se refiere, en especial, a la discriminación indirecta, en cuyo caso los datos estadísticos desempeñan a menudo un papel fundamental para probar los efectos adversos de una medida aparentemente neutral dirigida a un grupo específico. El primer informe sobre la aplicación de la Directiva 2000/43/CE alertó de que "la escasez de datos sobre el origen étnico de los trabajadores en la mayoría de los Estados miembros puede dificultar la correcta evaluación de la aplicación de la legislación comunitaria". La situación permanece prácticamente inalterada en lo que atañe a ambas Directivas (Quesada, 2008). Este problema ha sido señalado por muchos encuestados -organismos nacionales de promoción de la igualdad, Agencia de Derechos Fundamentales, ONG-, y la Comisión comparte sus preocupaciones.

La mayoría de los Estados miembros aceptan los datos estadísticos o test de situación como prueba de discriminación. Un ejemplo es el test de situación que es un método que pone de manifiesto la discriminación mediante una comparación por pares; por ejemplo, utilizando dos solicitudes idénticas que difieran solamente en una característica particular objeto de examen (por ejemplo, la edad).

No obstante, muchos Estados miembros no recogen datos sobre la igualdad de trato o lo hacen de forma muy limitada, amparándose en la normativa de protección de datos. Debe hacerse hincapié en que la legislación de la UE, en particular la Directiva sobre la protección de datos, no impide a los Estados miembros recabar datos para producir estadísticas, siempre que se respeten las garantías establecidas en la Directiva. Formular orientaciones prácticas o establecer normas para la recogida de datos sobre la igualdad de trato a nivel nacional es una buena práctica que parece ofrecer al menos un punto de partida a la hora de abordar este problema. Por ejemplo, Alemania, Irlanda, Francia, Croacia y Hungría han publicado estudios, informes o manuales sobre la recogida de datos sobre igualdad de trato.

Toda la información disponible confirma los bajos niveles de denuncia de casos de discriminación, tanto en la fase inicial, por ejemplo, a un organismo de promoción de la igualdad o a la policía, como en la fase de acción judicial.

Las tendencias más recientes muestran que entre todos los grupos étnicos e inmigrantes encuestados, el $82 \%$ de las personas que sufrieron discriminación no lo denunciaron. Entre las razones aducidas para no iniciar un procedimiento está la convicción de que la denuncia no prosperará, la falta de conocimiento sobre la forma y el destinatario de la misma, además de las experiencias negativas debidas a los inconvenientes, la burocracia o la duración del proceso. Contrariamente a ciertas inquietudes, expresadas antes de la adopción de las Directivas, no se ha registrado un aumento sustancial de los procesos judiciales en materia de discriminación. El número de casos denunciados es, por lo general, escaso y se estima que representa solamente un reducido porcentaje de los casos de discriminación que realmente tienen lugar en territorio comunitario. 
En algunos Estados miembros, las cifras pueden incluso ser demasiado bajas, debido a que los casos de discriminación manifiestos no se denuncian ni se procesa a sus autores. Este hecho pone de relieve la necesidad de redoblar los esfuerzos de concienciación e información, así como de mejorar el acceso a la justicia y a los mecanismos de denuncia (Torres e Izquierdo, 2010). Los organismos nacionales de promoción de la igualdad podrían desempeñar un papel importante para simplificar y facilitar la denuncia de las víctimas de discriminación (EQUINET, 2012).

El acceso a una justicia rápida y eficaz es de capital importancia para las víctimas de discriminación. Entre los obstáculos al acceso a la justicia, se encuentran los plazos breves para presentar una denuncia por discriminación, además de la duración y el coste de los procesos, incluido el posible efecto disuasorio que ejerce sobre las víctimas el principio de quien pierde, paga y la limitada disponibilidad de asistencia jurídica gratuita.

Las dos Directivas no armonizan las sanciones y los recursos en caso de discriminación, pero obligan a los Estados miembros a establecer sanciones efectivas, proporcionadas y disuasorias, y a garantizar que se puedan incoar acciones judiciales para el cumplimiento de las obligaciones establecidas en ellas, precedidas en su caso de un procedimiento administrativo contencioso.

Los problemas iniciales que muchos Estados miembros se plantearon en relación con la correcta transposición de las normas sobre sanciones ya han sido resueltos, y las sanciones previstas por la ley resultan, generalmente, apropiadas. Sin embargo, subsisten motivos de preocupación en lo que se refiere a la disponibilidad de recursos en la práctica, y a que las sanciones que se impongan a casos concretos cumplan plenamente con las disposiciones de ambas Directivas.

Los órganos jurisdiccionales nacionales parecen mostrar cierta tendencia a utilizar un baremo más laxo a la hora de aplicar las sanciones previstas en la ley, así como en el nivel y el importe de las indemnizaciones fijadas. En el caso de ACCEPT, el TJUE señaló que la Directiva 2000/43/CE impide que la ley nacional disponga de sanciones puramente simbólicas ya que, con arreglo a determinadas condiciones, se incumpliría la Directiva si únicamente cupiera la amonestación en caso de discriminación.

El TJUE ha aclarado en su jurisprudencia la interpretación de las dos Directivas. La mayoría de los asuntos se refieren a la interpretación de la Directiva 2000/78/CE en lo relativo a la discriminación por motivos de edad, y en particular al artículo 6, apartado 1, que establece que las diferencias de trato basadas en la edad pueden encontrar justificación si existe una finalidad legítima y si los medios empleados para alcanzar tal finalidad resultan apropiados y necesarios.

Por otra parte, la jurisprudencia relativa a la discriminación por motivos de orientación sexual, discapacidad, origen racial o étnico se encuentra menos desarrollada, ya que se han presentado menores procedimientos ante el TJUE. En los asuntos por estos motivos, el TJUE ha abordado cuestiones fundamentales, tales como la prohibición a un empleador de efectuar un anuncio general que implique discriminación, la definición de discapacidad o la exclusión de las parejas del mismo sexo de la percepción de prestaciones laborales reservadas a las parejas heterosexuales.

El TJUE todavía no ha tenido la oportunidad de pronunciarse sobre la discriminación por motivos de religión o de convicciones, aunque los Estados miembros ya informan sobre su aplicación en su jurisprudencia nacional. 


\section{TENDENCIA NORMATIVA Y EFECTOS CONVERGENTES}

Si bien existen algunas características específicas de cada motivo de discriminación, la estructura de las dos Directivas y los conceptos básicos son semejantes en ambos casos definiciones, acción positiva, características mínimas, defensa de derechos, carga de la prueba, difusión de la información, diálogo con los interlocutores sociales y ONG, sanciones.

La discriminación indirecta se da cuando una disposición, criterio o práctica aparentemente neutro sitúa a personas con una característica determinada en desventaja particular con respecto a otras personas, salvo que dicha disposición, criterio o práctica pueda encontrar justificación objetiva en una finalidad legítima y que los medios para la consecución de esta finalidad sean adecuados y necesarios. El concepto de discriminación indirecta es complejo y muchos Estados miembros se enfrentaron a dificultades iniciales en su correcta transposición. Ahora se halla consagrado en la legislación, pero su aplicación en la práctica aún supone un reto. Como ilustración de este problema, algunos Estados miembros informan de que en los tribunales nacionales han expresado su preocupación por la falta de claridad o de comprensión del concepto de discriminación indirecta. Otros Estados miembros señalan que todavía no disponen de jurisprudencia que facilite una interpretación de la discriminación indirecta. Los organismos de promoción de la igualdad, en su contribución común, apuntaron a una necesidad específica de supervisión a fin de garantizar la aplicación uniforme de las normas relativas a la discriminación indirecta (EQUINET, 2013).

Un elemento clave necesario para garantizar el correcto tratamiento de las denuncias por discriminación es la inversión de la carga de la prueba ante los tribunales y demás autoridades competentes. Esto significa que, cuando una persona alegue ser víctima de discriminación y pueda establecer los hechos de los que se pueda desprender la presunción de discriminación, corresponde a la parte demandada probar que no ha habido discriminación. Inicialmente ocho Estados miembros tuvieron problemas para la correcta transposición del concepto de carga de la prueba.

Algunos Estados miembros señalan que la aplicación correcta de la inversión de la carga de la prueba aún supone un reto y no es lo suficientemente conocida por los tribunales nacionales. Como ejemplo de una forma de solucionar este problema, un Estado miembro ha informado de que está considerando la inclusión de la inversión de la carga de la prueba directamente en las normas procesales civiles, y no únicamente en la legislación sobre la igualdad de trato. La Comisión Europea está fomentando la correcta aplicación de este concepto mediante la formación de los jueces nacionales y los profesionales de la justicia.

Las Directivas autorizan expresamente a los Estados miembros, sin obligarlos, a adoptar medidas específicas destinadas a prevenir o compensar las desventajas ocasionadas por cualquiera de los motivos contemplados en las Directivas. Se entiende que la acción positiva, por definición, debe procurar beneficios a los grupos destinatarios. Casi todos los Estados miembros han considerado algún tipo de acción positiva en el ámbito de aplicación de las dos Directivas, por ejemplo, en favor de las personas con discapacidad o de los romaníes.

Por lo que se refiere a las acciones positivas en favor de las personas con discapacidad, los Estados miembros determinan los objetivos de contratación de los empleadores del sector público. Según se informa, la acción positiva en favor de los romaníes es más diversa e incluye los cuatro sectores clave de las estrategias nacionales de integración de los romaníes empleo, vivienda, educación y salud-. Las variables cualitativas culturales son una fuente de condicionantes de la interpretación y aplicación de la normativa, asumir la diversidad cultural en la aplicación normativa se convierte en un objeto de estudio cuantificable, por consiguiente, un objeto susceptible de evaluación (Bhabha, 2002). 
En la Propuesta de la Comisión para una Recomendación del Consejo sobre las medidas de integración efectiva de los romaníes en los Estados miembros, adoptada el 9 de diciembre de 2013, documento del Consejo no 16970/13, se recomienda que "a fin de garantizar la plena igualdad en la práctica de los romaníes, los Estados miembros deben adoptar medidas específicas que aseguren la igualdad de trato y el respeto de los derechos fundamentales, incluida la igualdad de acceso a la educación, el empleo, la asistencia sanitaria y la vivienda".

Por otra parte, las Directivas no recogen ninguna disposición específica sobre las discriminaciones múltiples, pero ambas se refieren al hecho de que "a menudo, las mujeres son víctimas de discriminaciones múltiples". Ello supone la existencia de una laguna normativa que las ciencias sociales en general, y el trabajo social en particular, recomiendan compensar con un marco regulador específico (Hall, 2009).

Ambas Directivas se refieren asimismo a la necesidad de evaluar, de conformidad con el principio de integración de la dimensión de género, el impacto de las medidas sobre mujeres y hombres -artículo 17, apartado 2, de la Directiva 2000/43/CE y artículo 19, apartado 2, de la Directiva 2000/78/CE-.

El TJUE ha decidido en diversas sentencias que, en determinadas circunstancias, la discriminación por discapacidad puede incluir la discriminación basada en la asociación del demandante con una persona que padezca una discapacidad, aunque este no la tenga. Este razonamiento parece ser de índole general y aplicable también a los demás motivos de discriminación regulados por las dos Directivas.

De la jurisprudencia nacional, se desprende que la Comisión considera que las Directivas también prohíben las situaciones en las que una persona es víctima de discriminación directa debido a una suposición o percepción errónea de características protegidas; por ejemplo, si un candidato no es seleccionado porque el empresario indebidamente considera que es de un determinado motivo religioso, origen étnico u homosexual (Torres y Fernández, 2012).

Las dos Directivas dejan claro que la prohibición de discriminación se aplicará asimismo a los nacionales de terceros países, pero no se contemplan las diferencias de trato basadas en la nacionalidad, y ello se entiende sin perjuicio de las disposiciones que regulan la entrada y la residencia. Se trata de un elemento importante de ambas Directivas que pone de relieve que la prohibición de discriminación protege a todas las personas que se encuentren en territorio de la Unión Europea y no solo a los ciudadanos de la UE.

Los ejemplos de Directivas que establecen de forma específica derechos de la igualdad de trato de los nacionales de terceros países son los siguientes:

Directiva 2003/109/CE del Consejo, de 25 de noviembre de 2003, relativa al estatuto de los nacionales de los terceros países residentes de larga duración (DO L 16, 23.1.2004, p. 44), modificada por la Directiva 2011/51/UE (DO L 132, 19.5.2011, p.1) con el fin de extender su ámbito de aplicación a los beneficiarios de protección internacional.

Directiva 2011/98/UE del Parlamento Europeo y del Consejo, de 13 de noviembre de 2011, por la que se establece un procedimiento único de solicitud de un permiso único que autoriza a los nacionales de terceros países a residir y trabajar en el territorio de un Estado miembro y por la que se establece un sino de cómo se aplica la legislación pertinente en la práctica. La legislación también debe combinarse con medidas políticas y financieras adecuadas. Los principios básicos comunes sobre la integración crean un marco para la cooperación política en la UE en la integración de nacionales de terceros países, incluido el respeto de la igualdad y la no discriminación, que se ha ido desarrollando a través de las Comunicaciones de la Comisión sobre la integración, los intercambios entre los Estados miembros y la consulta con las partes interesadas pertinentes. 
Los Estados miembros informan de que la protección contra la discriminación se aplicará a todas las personas que vivan en un país determinado cuando ésta tenga lugar por motivos recogidos en las dos Directivas. Varios Estados miembros describen sus políticas de integración de los inmigrantes y los nacionales de terceros países como una labor de prevención contra la discriminación.

\section{CONCLUSIONES}

Los Estados miembros han adoptado las medidas necesarias para transponer las dos Directivas en sus respectivos ordenamientos jurídicos nacionales, así como para establecer los procedimientos y los organismos imprescindibles para la aplicación de estas Directivas.

Las autoridades administrativas y judiciales de los Estados miembros, así como sus organismos de promoción de la igualdad, han desarrollado sus capacidades de intervención social y jurídica en una orientación garantista y sistemática hacia la plena protección en la práctica. Sin embargo, aún persisten asimetrías de interpretación con incidencias reduccionistas en la aplicación normativa.

El auge y expansión de la islamofobia (Torres y Fernández, 2013) permite inferir que los futuros perfeccionamientos normativos de estas directivas se deberían dirigir a esta manifestación específica de la discriminación por motivos religiosos. Sin embargo, debe acometerse el requisito previo de llevar a cabo estudios desde los ámbitos de la ciencias jurídicas, de la administración y desde el área de conocimiento específica del trabajo social (Torres e Izquierdo, 2012).

En el ámbito comunitario, la prioridad de la Comisión Europea reside en aumentar la concienciación sobre la protección ya existente, a fin de garantizar una mejor aplicación y ejecución prácticas de las Directivas, pues la legislación por sí sola no es suficiente para garantizar la plena igualdad, por lo que debe combinarse con medidas políticas adecuadas. En este sentido, se deben reforzar la cooperación entre la Unión y los Estados miembros en el ámbito del Programa de la Unión Europea para el empleo y la solidaridad social (Progress) con objeto de garantizar mejoras tangibles en la concienciación del conjunto de derechos desplegados en el territorio común. También, consideramos que reforzar el papel de los organismos nacionales de promoción de la igualdad, como garantes de la igualdad, puede contribuir decisivamente a una aplicación y ejecución más eficaz de las Directivas.

Finalmente, aumentar la eficacia de los organismos de promoción de la igualdad y permitirles desarrollar todo su potencial es una medida prioritaria para promover la igualdad de trato, de modo que ésta resulte fácilmente accesible a todos los ciudadanos de la Unión, pues es más rápida y menos costosa que la vía judicial para todas las partes interesadas, incluidos los Estados miembros.

\section{BIBLIOGRAFÍA}

BAMFORTH, N.; MALIK, M. y O'CINNEIDE, C. (2008). Discrimination Law: Theory and Context. Londres: Sweet and Maxwell.

BESSON, S. (2006). "The European Union and Human Rights: Towards a Post National Human Rights Institution". Human Rights Law Journal, 6 (2): 323-360.

BHABHA, H.K (2007). La ubicación de la Cultura. Buenos Aires: Ediciones Manatial.

CHALMERS, D.; DAVIES, G. y MONTI, G. (2010). European Union law: cases and materials. Cambridge: University Press. 
CONSEJO DE LA UNIÓN EUROPEA (2010). Directiva 2000/43/CE de 29 de junio de 2000, relativa a la aplicación del principio de igualdad de trato de las personas independientemente de su origen racial o étnico. DOCE ${ }^{\circ}$ L 180/22, de 19 de julio de 2000.

CONSEJO DE LA UNIÓN EUROPEA (2010). Directiva 2000/78/CE, de 27 de noviembre de 2000, relativa al establecimiento de un marco general para la igualdad de trato en el empleo y la ocupación. DOCE ${ }^{\circ} \mathrm{L} 303$ de 2 de diciembre de 2000.

HALL, S. (2000). "Une perspective européenne sur l'hybridation: éléments de réflexion". Hermès, La Revue, (3): 99-102.

INFORME EQUINET (2012). Equality law in Practice. Report on the Implementation of the Race and General Framework Directives. Bruselas: Equinet, 2013, en: http://www.equineteurope.org/Report-onthe-Implementation-of

INFORME EQUINET (2012). Tackling the 'Known Unknown' How Equality Bodies Can Address Under-Reporting of Discrimination through Communications. Bruselas: Equinet, 2012, en: $\mathrm{http} / / / \mathrm{www}$.equineteurope.org/IMG/pdf/tackling_the_known_unknown_final_merged_pdf

SEGURA, R. Q. (2007). "La no discriminación, la igualdad de trato y de oportunidades, en el ordenamiento europeo. Del Convenio Europeo de Derechos Humanos del Consejo de Europa, a los Tratados ya la Carta de los Derechos Fundamentales de la Unión Europea". Revista del Ministerio de Trabajo e Inmigración y Asuntos Sociales, 3: 53-75.

TORRES R.D. y FERNÁNDEZ, T. (2012). "Musulmanas en Europa y las prácticas discriminatorias y restrictivas en la educación". Revista Española del Tercer Sector, 22: 151-186.

TORRES R. D. y FERNÁNDEZ, T. (2012). "Perspectiva Hermenéutica y Epistemológica para el Trabajo Social Comunitario con Mujeres Musulmanas". Revista Sistema, 229: 57-72.

TORRES R. D. e IZQUIERDO, J.D. (2012). "Islamofobia en Europa: La intervención social comunitaria fundada en la cosmovisión del Análisis Transaccional". Revista de Análisis Transaccional y Psicología Humanista, 67: 207-297.

TORRES R. D. e IZQUIERDO, J.D. (2012). "Las intervenciones belga y francesa contra las prácticas discriminatorias en el empleo de personas musulmanas consideradas referentes para España". Revista de Derecho Social, 2012: 107-210.

\section{Breve currículo:}

\section{Jesús Pérez Viejo}

Licenciado en Psicología, Doctor cum laude en Trabajo Social por la Universidad de Castilla La Mancha, Máster Universitario en Intervención Social en las Sociedades del Conocimiento por la Universidad UNIR. Colabora con la Universidad Internacional de La Rioja. Ha sido profesor Asociado de la UNED y de la Universidad Carlos III, docente de diferentes Máster universitarios y con amplia experiencia en la formación de profesionales de entidades públicas y privadas en temas relacionados con la intervención social, la violencia, el counselling, las habilidades de relación de ayuda y los autocuidados en equipos profesionales, a nivel nacional e internacional.

\section{César Muñoz Martínez}

Licenciado en Economía y en Derecho por la Universidad Carlos III de Madrid, Doctor en Unión Europea en la UNED (Premio Extraordinario), donde desempeño la función de Profesor en la unidad docente de Unión Europea del Departamento de Economía Aplicada. Mi principal línea de investigación es la Economía de Transporte, donde he publicado varias investigaciones relacionadas con el binomio instituciones comunitarias e infraestructuras de transporte. Adicionalmente, he profundizado en otros ámbitos de las ciencias sociales como el sector turístico y distintas políticas sectoriales comunitarias. 